\title{
Ambient pressure sensitivity of microbubbles investigated through a parameter study
}

\section{Andersen, Klaus Scheldrup; Jensen, Jørgen Arendt}

Published in:

Journal of the Acoustical Society of America

Link to article, DOI:

$10.1121 / 1.3242359$

Publication date:

2009

Document Version

Publisher's PDF, also known as Version of record

Link back to DTU Orbit

Citation (APA):

Andersen, K. S., \& Jensen, J. A. (2009). Ambient pressure sensitivity of microbubbles investigated through a parameter study. Journal of the Acoustical Society of America, 126(6), 3350-3358.

https://doi.org/10.1121/1.3242359

\section{General rights}

Copyright and moral rights for the publications made accessible in the public portal are retained by the authors and/or other copyright owners and it is a condition of accessing publications that users recognise and abide by the legal requirements associated with these rights.

- Users may download and print one copy of any publication from the public portal for the purpose of private study or research.

- You may not further distribute the material or use it for any profit-making activity or commercial gain

- You may freely distribute the URL identifying the publication in the public portal

If you believe that this document breaches copyright please contact us providing details, and we will remove access to the work immediately and investigate your claim 


\title{
Ambient pressure sensitivity of microbubbles investigated through a parameter study
}

\author{
Klaus Scheldrup Andersen and Jørgen Arendt Jensen \\ Department of Electrical Engineering, Center for Fast Ultrasound Imaging, Technical University \\ of Denmark, Building 348, 2800 Kgs. Lyngby, Denmark
}

(Received 5 February 2009; revised 31 August 2009; accepted 8 September 2009)

\begin{abstract}
Measurements on microbubbles clearly indicate a relation between the ambient pressure and the acoustic behavior of the bubble. The purpose of this study was to optimize the sensitivity of ambient pressure measurements, using the subharmonic component, through microbubble response simulations. The behavior of two microbubbles corresponding to two different contrast agents was investigated as a function of driving pulse and ambient overpressure $p_{\text {ov }}$. Simulations of Levovist using a rectangular driving pulse show an almost linear reduction in the subharmonic component as $p_{\mathrm{ov}}$ is increased. For a 20 cycle driving pulse, a reduction of $4.6 \mathrm{~dB}$ is observed when changing $p_{\mathrm{ov}}$ from 0 to $25 \mathrm{kPa}$. Increasing the pulse duration makes the reduction even clearer. For a pulse with 64 cycles, the reduction is $9.9 \mathrm{~dB}$. This simulation is in good correspondence with measurement results presented in the literature. Further simulations of Levovist show that also the shape and the acoustic pressure of the driving pulse are very important factors. The best pressure sensitivity of Levovist was found to be $0.88 \mathrm{~dB} / \mathrm{kPa}$. For Sonazoid, a sensitivity of $1.14 \mathrm{~dB} / \mathrm{kPa}$ has been found, although the reduction is not completely linear as a function of the ambient pressure.
\end{abstract}

(C) 2009 Acoustical Society of America. [DOI: 10.1121/1.3242359]

PACS number(s): 43.80.Vj, 43.25.Yw [CCC]

Pages: $3350-3358$

\section{INTRODUCTION}

Local blood pressure measurements provide important information on the state of health of the organs in the human body and can be used to diagnose severe heart, lung, and kidney diseases. The pressure is currently measured locally in arteries and organs by means of a pressure catheter. As this is an invasive technique, it is inconvenient to the patient, there is a risk of infection, and the catheter will inevitably introduce changes to the blood flow and thereby the pressure. Therefore, many attempts to find a noninvasive procedure have been made. When evaluating new approaches for noninvasive local blood pressure measurements, the sensitivity is a crucial factor. Although the human blood pressure varies between 0 and approximately $25 \mathrm{kPa}(1 \mathrm{kPa}=7.5 \mathrm{~mm} \mathrm{Hg})$, it should still be possible to distinguish pressure differences as low as $1-3 \mathrm{kPa}$ to measure the blood pressure in the small veins and arteries. One noninvasive approach that has been suggested is to perform Doppler echocardiography using a simplified modification of the Bernoulli equation. ${ }^{1,2}$ This method was, however, concluded not to yield reproducible or reliable results by Strauss et al. ${ }^{3}$ Another type of approach, which is still being investigated, is to combine microbubbles injected into the blood and diagnostic ultrasound.

Bubbles in a fluid can be used for measurement of pressure gradients due to their size dependent oscillations. ${ }^{4-6}$ Since the introduction of ultrasound contrast agents (UCAs), many approaches on how to exploit their ambient pressure sensitivity have been presented. One of the first to propose noninvasive measurement of cardiac pressure using an UCA were Fairbank and Scully ${ }^{4}$ in 1977. They claimed that the acoustic properties of the microbubbles change when the size of the bubbles changes. To measure these changes, they sug- gested the use of resonance excitation. However, due to the large size distribution of the first generation UCAs containing free bubbles, their results were inconclusive. Other suggestions from that time are by $\mathrm{Hok}^{5}$ in 1981 and Shankar et $a l^{6}$ and Newhouse and Shankar ${ }^{7}$ in 1986. Newhouse and Shankar showed theoretically and experimentally that accurate bubble size measurements are possible using a double frequency technique for determination of the sum and difference frequencies. The rapid dissolution time after injection of the free air bubbles prevented, however, any practical implementation.

With the introduction of the more stable second generation UCAs, the circulation system can now be used to transport the encapsulated microbubbles to the region of interest. This has initiated new attempts to exploit the ambient pressure dependent acoustic properties that the high compressible air and gas bubbles possess. Bouakaz et al. ${ }^{8}$ presented in 1999 an approach for measuring the disappearance time of free bubbles, which were generated at the region of interest by rupturing the contrast agent microbubbles using a lowfrequency high acoustic amplitude pulse. From in vitro experiments they concluded the approach to have a resolution of $6.7 \mathrm{kPa}(50 \mathrm{~mm} \mathrm{Hg})$. Later, they suggested that the resolution could be improved by using larger bubbles or by using wavelet processing or a combination of this. ${ }^{9}$ No in vivo results or further investigations have, however, been presented using this approach yet. Around the same time, Shi et $a l .{ }^{10}$ observed from experiments that the subharmonic component of Levovist is highly sensitive to ambient pressure changes compared to the fundamental and the second harmonic component. They reported a $9.9 \mathrm{~dB}$ linear decrease in the peak amplitude of the subharmonic component when increasing the ambient hydrostatic pressure from 0 to $24.8 \mathrm{kPa}$ 
$(186 \mathrm{~mm} \mathrm{Hg})$. Furthermore, they found that the ambient pressure-induced reduction was highest when the acoustic excitation pressure was around the growth stage of the subharmonic, which occurs when the acoustic driving pressure causes the subharmonic component to increase rapidly from background noise level to be clearly visible in the spectrum. Recently, the same group has presented similar results for Sonazoid, which was found to have an average decrease of $13.3 \mathrm{~dB} .{ }^{11}$ Furthermore, in 2005 the same group presented in vivo results for proof of concept of the capabilities of the subharmonic response. ${ }^{12}$ As the measurements were performed directly on the abdominal cavity and the aorta by incision of two dogs, this can hardly be characterized as noninvasive. However, the results still showed that the subharmonic component decreased as the ambient pressure increased and thereby indicated that the subharmonic response of UCAs can be used for ambient pressure measurements. Also in 2005, Adam et al. ${ }^{13}$ investigated microbubbles' response to cyclic ambient pressure changes by mimicking left ventricular pressure changes. They found that the subharmonic response correlated best with the cyclic changes compared to the fundamental and second harmonic, but also observed a transient delay before this correlation occurred. In 2008, Andersen and Jensen ${ }^{14}$ presented a new experimental setup, which more realistically resembles a clinical setting using a single array transducer. The setup was used to measure the ambient pressure sensitivity of SonoVue and confirmed the previous findings, revealing a pressure sensitivity of $0.42 \mathrm{~dB} / \mathrm{kPa}$. The same group has also investigated the dependence on the acoustic driving pressure experimentally. ${ }^{15}$ However, the driving pressure was selected too high causing bubble destruction, and the investigation was therefore inconclusive.

Modeling the acoustics of bubbles in a fluid is a still ongoing investigation, which was initiated by Rayleigh ${ }^{16}$ in 1917 who studied damages to ship propellers due to bubble cavitation. In 1933, Minnaert ${ }^{17}$ explained the characteristic resonance frequency of free bubbles when he did a theoretical and experimental study of bubbles' emission of sound. Since then, several modifications to the existing models and new theoretical models on how to predict the behavior of an oscillating bubble have been presented. Most models are based on modifications of the Rayleigh-Plesset ${ }^{18}$ (RP) equation and are capable of handling shell encapsulating bubbles. This includes the models used by de Jong and Hoff ${ }^{19}$ and Church. ${ }^{20}$ Other models are based on the modified Herring equation to describe the radial motion (e.g., Morgan et al. ${ }^{21}$ ). Recently, Vos et al. ${ }^{22}$ proposed a novel approach for investigation of full populations of microbubbles' behavior in acoustic fields, also based on the modified Herring equation. The study furthermore included a new method to estimate the viscoelastic shell properties of UCAs. Existing methods for this based on the Rayleigh-Plesset equation have been suggested by de Jong and $\operatorname{Hoff}^{19}$ and de Jong et al. ${ }^{23}$ and described further by Hoff. ${ }^{24}$

Despite the growing number of experiments within hydrostatic pressure measurements, no real parameter study investigating the response of microbubbles in respect to ambient pressure changes has been performed until now. The purpose of this study is to optimize the sensitivity of pressure measurements through bubble response simulations investigating the complex mechanisms for subharmonic generation. This is carried out by an extensive number of simulations of two commercial UCAs. Since the study focused on the effect of the driving pulse, the parameters of the microbubbles are fixed in all simulations, whereas several different settings regarding the excitation pulse were varied. Some part of this work has been presented at the 2008 SPIE Medical Imaging Symposium. ${ }^{25}$

This paper is organized as follows. Section II presents the choice of simulation model and parameters used for the investigation. Furthermore, it also describes the processing of the simulated response. The achieved results are presented and discussed in Sec. III. Finally, the investigation is summarized by a conclusion in Sec. IV.

\section{THEORY AND METHOD}

The investigation has been performed using the MATLAB (The Math Works Inc., Natick, MA) environment. To carry out the simulations, the free simulation program BUBBLESIM by $\mathrm{Hoff}^{24}$ is used. BUBBLESIM is a toolbox that calculates the oscillation and scattered echo for a specified contrast agent microbubble and excitation pulse. It numerically solves a second order ordinary differential equation (ODE) that has been combined from a set of equations, each equation modeling different parts (bubble, shell, and surrounding liquid) of the system that makes up a contrast agent microbubble. In BUBBLESIM, the following four different models are implemented: The $\mathrm{RP}^{18}$ model, the Trilling ${ }^{26}$ model, the Keller-Miksis ${ }^{27}$ model, and a modified version of the RP model, which is an intermediate model of the RP on one hand and the Trilling and Keller-Miksis models on the other hand. The largest disadvantage of the RP model is that it does not include radiation damping, which is energy loss caused by radiation of sound. This is accounted for in the Trilling and the Keller-Miksis models, which both include a finite but constant speed of sound in the liquid. However, both the Trilling and Keller-Miksis models have a risk of becoming numerically unstable when the bubble wall velocity becomes comparable to the speed of sound (acoustic Mach numbers, $M=\dot{R} / c$, around unity). This happens for high oscillation amplitudes and causes the models to have an unphysical negative inertia. Instead, Hilgenfeldt and Lohse ${ }^{28}$ suggested a modified version of the RP model that includes the radiation damping term from the Trilling and KellerMiksis models. This is also used in the modified version implemented in BUBBLESIM, which is the model selected for the parameter investigation. It was chosen because of its numerical stability, which is important when doing many simulations spanning a wide range of variable changes. The model is based on the theoretical description of microbubbles as air-filled particles with surface layers of elastic solids presented by Church $^{20}$ and later derived by $\operatorname{Hoff}^{24}$ to describe a polymeric microbubble: 


$$
\begin{aligned}
\rho_{L} a \ddot{a}+\frac{3}{2} \rho_{L}(\dot{a})^{2}= & p_{G e}\left(\frac{a_{e}}{a}\right)^{3 \kappa}-p_{\mathrm{ov}}-p_{\mathrm{ac}}(t)-4 \mu_{L} \frac{\dot{a}}{a} \\
& -12 \mu_{S} \frac{d_{S e} a_{e}^{2}}{a^{3}} \frac{\dot{a}}{a}-12 G_{S} \frac{d_{S e} a_{e}^{2}}{a^{3}}\left(1-\frac{a_{e}}{a}\right) .
\end{aligned}
$$

In Eq. (1), $\rho_{L}$ is the surrounding liquid density, $a(t)$ and $a_{e}$ are the instantaneous and equilibrium outer bubble radii, respectively, $\dot{a}=d a / d t$ denotes derivation with respect to time $t, p_{G e}$ is the pressure in the gas inside the bubble at equilibrium, $\kappa$ is the polytropic exponent of the gas, $p_{\mathrm{ov}}$ is the hydrostatic pressure in the surrounding liquid, $p_{\mathrm{ac}}(t)$ is the driving acoustic pressure, $\mu_{L}$ and $\mu_{S}$ are the shear viscosities in the liquid and shell, respectively, $d_{S e}$ is the shell thickness, and $G_{S}$ is the shell shear modulus. Rewriting Eq. (1) in a simplified form gives

$$
\rho_{L} a \ddot{a}+\frac{3}{2} \rho_{L}(\dot{a})^{2}=p_{L}-p_{\mathrm{ov}}-p_{\mathrm{ac}}(t),
$$

where

$$
p_{L}=p_{G e}\left(\frac{a_{e}}{a}\right)^{3 \kappa}-4 \mu_{L} \frac{\dot{a}}{a}-\Delta T_{S}
$$

describes the pressure at the bubble surface and

$$
\Delta T_{S}=12 \frac{d_{S e} a_{e}^{2}}{a^{3}}\left(G_{S}\left(1-\frac{a_{e}}{a}\right)+\mu_{S} \frac{\dot{a}}{a}\right)
$$

denotes the stress across the shell. The left hand side of Eq. (2) represents the inertia of the liquid due to the oscillating bubble while the right hand side denotes the damping viscous and restoring stiffness forces. The pressure in the gas at equilibrium $p_{G e}$ is equal to the hydrostatic pressure in the liquid and a term caused by surface tension. ${ }^{20}$ However, to prevent immediate bubble shrinkage upon formation, the shell strain is assumed to counteract the latter part; hence $p_{G e}=p_{\mathrm{ov}}$. Finally, from Eq. (4) it is seen that the model requires the viscous and elastic properties of the shell described by $\mu_{S}$ and $G_{S}$. These must, however, be estimated experimentally as described below.

Any numerical solver can be used to solve the ODE in Eq. (1). In this study, the solver of variable order from 1 to 5 has been selected as it should be more reliable and stable for solving situations where the differential equation becomes stiff. $^{24}$ This occurs, for example, when the bubble radius changes slowly during the expansion phase but goes through very fast changes in radius and velocity under compression. The choices on simulation model and numerical solver, as well as other general setup parameters, for the simulations in this study are summarized in Table I.

In this study, a batch mode for BUBBLESIM has been created as this gives a bit more control and, more importantly, it makes it possible to perform multiple simulations automatically, which is essential in a parameter study like this. Furthermore, one modification has been made to BUBBLESIM. In its original form, it is not possible to change the ambient overpressure parameter denoted by $p_{\mathrm{ov}}$ in Eq. (1). Since this is crucial when investigating microbubbles' sensi-
TABLE I. List of simulation parameters regarding the general setup of BUBBLESIM.

\begin{tabular}{lc}
\hline \hline Parameter & Designation \\
\hline ODE solver & ODE15s \\
Simulation model & Modified Rayleigh-Plesset \\
Thermal damping & Isothermal \\
Liquid & Water \\
\hline \hline
\end{tabular}

tivity to ambient pressure changes, this feature has been enabled by small modifications to the source code.

While the bubble size distribution can be determined with a multisizer, it is somewhat more difficult to specify the elastic and viscous shell parameters. One way to do this is to perform a combination of experiments and model fitting as described by Hoff and co-workers. ${ }^{19,23,24}$ This will, however, only give an estimate of proper designations, and usually an interval for some of the parameters is given. The procedure has been used by $\mathrm{Yu}$ et al. $^{29}$ and Hoff ${ }^{24}$ to estimate suitable parameters for the commercial contrast agents Levovist (Schering AG, Berlin, Germany) and Sonazoid (GE Healthcare, Oslo, Norway), respectively. These values used in the investigation were fixed for all simulations and are listed in Table II. Before a simulation can be carried out, a driving pulse must be selected. Since the emphasis of this study was to optimize the subharmonic sensitivity to ambient pressure changes as a function of the excitation pulse, a large number of different driving pulses were examined. The driving pulse was generated based on four different characteristics being the center frequency $f_{c}$, the number of pulse cycles $N_{c}$, the maximum acoustic pressure $p_{\mathrm{ac}}$, and the shape of the pulse. The possible designations used for the investigation are listed in the upper part of Table III. The center frequency was selected based on a preliminary study optimizing the energy of the subharmonic component to the fundamental, as shown for Sonazoid in Fig. 1. Figure 1(a) shows the energy of the subharmonic component as a function of the driving frequency. As can be seen, the energy peaks and is approximately the same for all driving frequencies displayed. Looking at the ratio of the subharmonic energy to the energy of the fundamental component in Fig. 1(b), however, shows that the subharmonic component is greater than the fundamental at two of the displayed frequencies. Therefore, $f_{c}$ $=2.46 \mathrm{MHz}$ was selected as the driving frequency when simulating the Sonazoid microbubble. As can be seen from Table III, 30 different settings for the acoustic pressure are used. This was decided to ensure determination of the growth stage of the subharmonic component with a reasonable precision. Although an acoustic pressure of $950 \mathrm{kPa}$ will prob-

TABLE II. List of the parameters from Yu et al. (Ref. 29) and Hoff (Ref. 24) used to describe the two different types of bubbles for the simulations in BUBBLESIM.

Contrast Bubble radius Shell thickness Shear modulus Shear viscosity

\begin{tabular}{lcccl} 
agent & $(\mu \mathrm{m})$ & $(\mathrm{nm})$ & $(\mathrm{MPa})$ & $(\mathrm{Pa} \mathrm{s})$ \\
\hline Levovist & 3.0 & 6.0 & 80 & 1.3 \\
Sonazoid & 3.2 & 4.0 & 52 & 0.99 \\
\hline \hline
\end{tabular}


TABLE III. List of parameters used in combination with the contrast agents listed in Table II. Combining all settings gives 3600 simulations in total for each agent.

\begin{tabular}{|c|c|c|c|c|c|c|c|c|c|c|c|}
\hline \multirow{2}{*}{$\begin{array}{l}\text { Parameter } \\
f_{c}\end{array}$} & \multicolumn{10}{|c|}{ Designation } & \multirow{2}{*}{$\begin{array}{c}\text { Unit } \\
\mathrm{MHz}\end{array}$} \\
\hline & 2.06 & 2.46 & & & & & & & & & \\
\hline$N_{c}$ & 1 & 2 & 5 & 10 & 20 & 32 & 48 & 64 & 128 & 256 & cycles \\
\hline \multirow[t]{3}{*}{$p_{\mathrm{ac}}$} & 100 & 150 & 200 & 250 & 275 & 300 & 325 & 350 & 375 & 400 & $\mathrm{kPa}$ \\
\hline & 425 & 450 & 475 & 500 & 550 & 575 & 600 & 650 & 675 & 700 & \\
\hline & 725 & 750 & 775 & 800 & 825 & 850 & 875 & 900 & 925 & 950 & \\
\hline \multicolumn{12}{|c|}{ Pulse shape "Rectangular" "Hanning" } \\
\hline$p_{\mathrm{ov}}$ & 0 & 5 & 10 & 15 & 20 & 25 & & & & & $\mathrm{kPa}$ \\
\hline
\end{tabular}

ably destroy the microbubbles in real measurements, the high values were selected to cover the entire range of subharmonic growth and saturation. The bottom row of Table III lists the designations of the ambient overpressures which were used in the simulations. As can be seen, the range covers the interval between 0 and $25 \mathrm{kPa}$ in steps of $5 \mathrm{kPa}$. In this way, the most common human blood pressure values are covered. Combining all the parameters in Table III gives a total of 3600 different simulations for each contrast agent.

When BUBBLESIM has completed a simulation, the simulated scattered pressure calculated $1 \mathrm{~m}$ from the center of the bubble is returned and the Fourier transformation is applied. Next, a search for the fundamental $\left(f_{0}\right)$, the first subharmonic $\left(\frac{1}{2} f_{0}\right)$, and the second harmonic $\left(2 f_{0}\right)$ component is performed, and the energy of each component is calculated. The center frequencies of the harmonic bands were selected as multiples of the emitted center frequency $f_{c}$. It should, however, be noted that initial simulations show that the frequency of the subharmonic component shifts slightly as the acoustic driving pressure is increased. The energy has been chosen over the peak amplitude since this is a more robust measure. The bandwidth to calculate the energy within was selected as the $-10 \mathrm{~dB}$ bandwidth of the excitation pulse.

\section{RESULTS AND DISCUSSION}

This section presents the results obtained through the simulation study. First, the fundamental, subharmonic, and

(a)

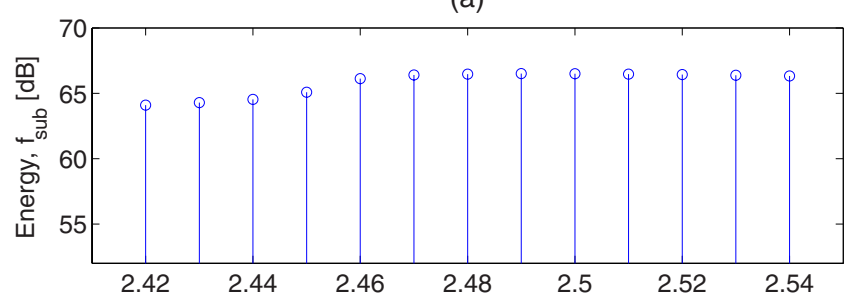

(b)

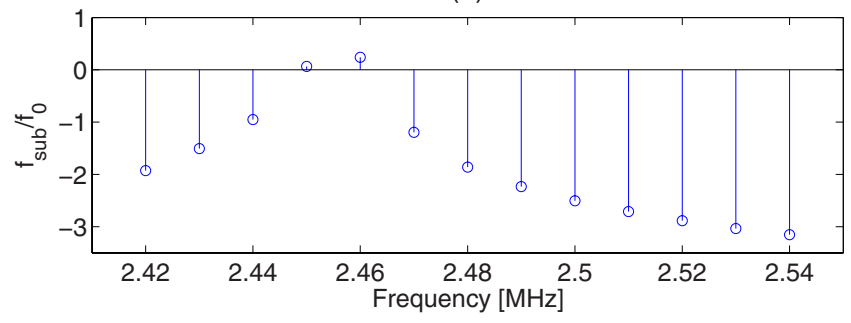

FIG. 1. (Color online) (a) shows the energy of the subharmonic component as a function of emitted frequency, while (b) shows the relation of the subharmonic to the fundamental component. second harmonic dependences on acoustic pressure will be presented. This is a natural step for two reasons. First of all, generation of the subharmonic component must be ensured before looking into the ambient pressure dependency. Another reason is to see at which acoustic pressures the growth stage of the subharmonic occurs for the two types of microbubbles. Along with this investigation, the scattered responses and spectra have been examined to ensure useful responses and proper selection of the bandwidth intervals to calculate the energy of the respective frequency components within. Since these results are rather trivial and take up a lot of space, only a few selected examples are presented in this section. In Sec. III B, the influence of ambient overpressure will be examined.

\section{A. Dependence on acoustic pressure}

Figure 2 shows the energy of the subharmonic, fundamental, and second harmonic components of Sonazoid as a function of acoustic pressure when a rectangular driving pulse for a different number of cycles is used. Each curve has been normalized by $88 \mathrm{~dB}$, which corresponds to the maximum energy observed among all simulations for both agents. Examining the subharmonic component, three characteristic
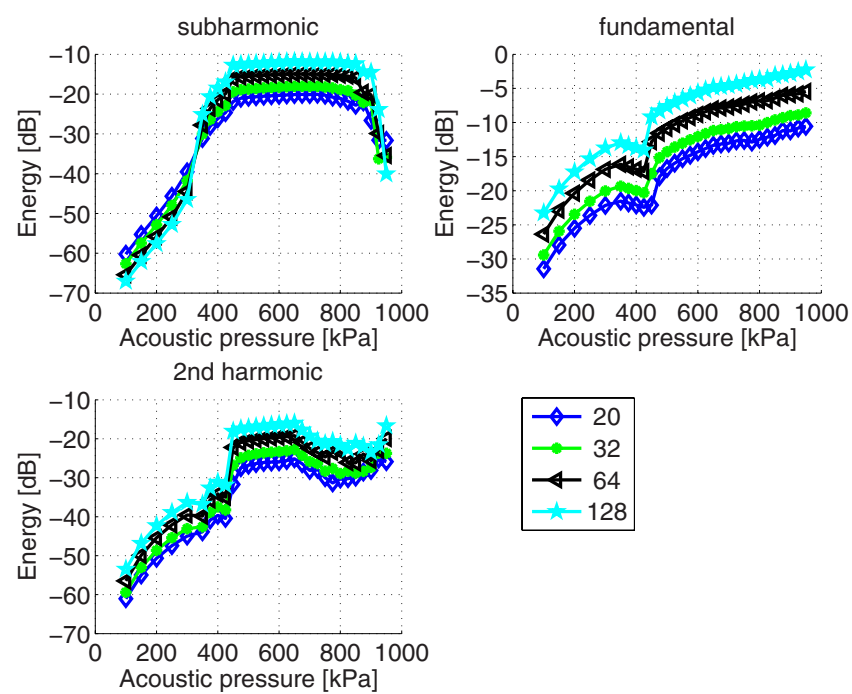

FIG. 2. (Color online) Energy as a function of acoustic pressure for Sonazoid. The driving pulse is a rectangular shaped sinusoid. Upper left graph shows the subharmonic behavior, upper right shows the first harmonic, and lower left presents the behavior of the second harmonic component. Each curve represents a different number of cycles in the driving pulse, as displayed in the legend to the lower right in the figure. 


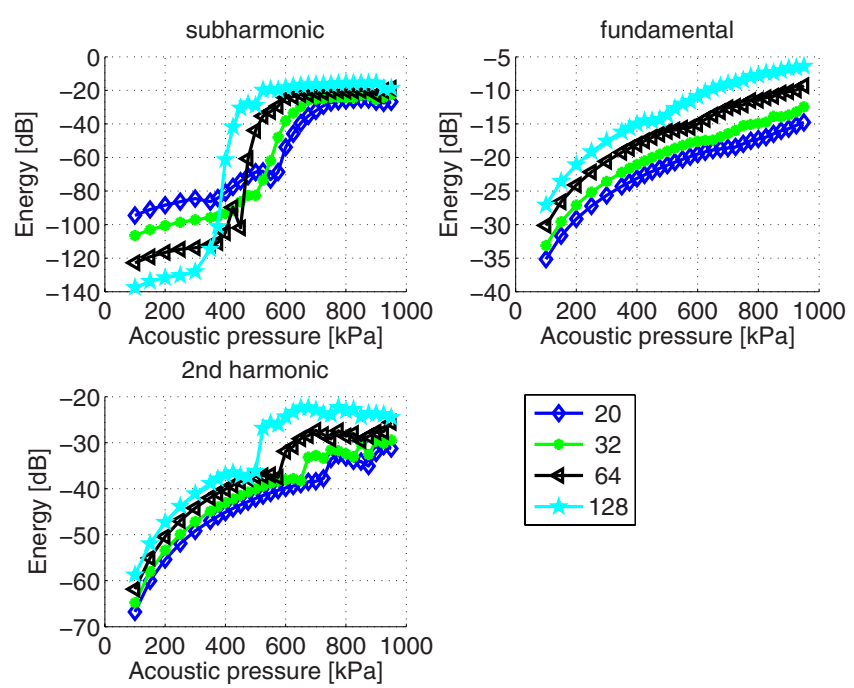

FIG. 3. (Color online) Energy as a function of acoustic pressure for Sonazoid. The driving pulse is a Hanning shaped sinusoid. Upper left graph shows the subharmonic behavior, upper right shows the first harmonic, and lower left presents the behavior of the second harmonic component. Each curve represents a different number of cycles in the driving pulse, as displayed in the legend to the lower right in the figure.

stages are clearly observed. In the occurrence stage for acoustic pressures below $300 \mathrm{kPa}$, the subharmonic is weak compared to the other components. For acoustic pressures in the interval between 300 and $425 \mathrm{kPa}$, the subharmonic increases rapidly, and this part can be characterized as the growth stage. This interval is in good agreement with measurements by Sarkar et al. ${ }^{30}$ who investigated the scattered response of Sonazoid at different driving pressures and frequencies. When increasing the acoustic pressure further, the growth eases off and can be compared to the saturation stage observed in measurements. Finally, when the acoustic pressure exceeds $875 \mathrm{kPa}$ the energy decreases again. At these levels, the corresponding spectra look noisy, indicating a stage of chaos. The pattern is the same for pulses of other lengths than displayed here, although the subharmonic component cannot be distinguished from the fundamental for driving pulses smaller than 5 cycles. The chaotic behavior at high acoustic pressure levels predicted in the simulations is actually in good correspondence with experimental results of free bubbles achieved by Lauterborn and Cramer. ${ }^{31}$ Looking at the fundamental, it increases almost linearly as expected. However, a slight drop is seen in the pressure interval corresponding to the growth stage of the subharmonic.

When the shape of the driving pulse is changed by applying a Hanning window, especially the subharmonic and second harmonic change behavior, as can be seen in Fig. 3. Regarding the subharmonic component, the three stage pattern is the same as observed for the rectangular driving pulse, although the interval of the growth period seems to have increased. This makes sense since less energy is transmitted using a Hanning shaped driving pulse compared to a rectangular signal of the same acoustic strength. Another interesting observation is that the acoustic pressure interval of the growth stage now is more dependent on the length of the driving pulse. The same pulse length dependent behavior is also seen for the second harmonic component. The funda-
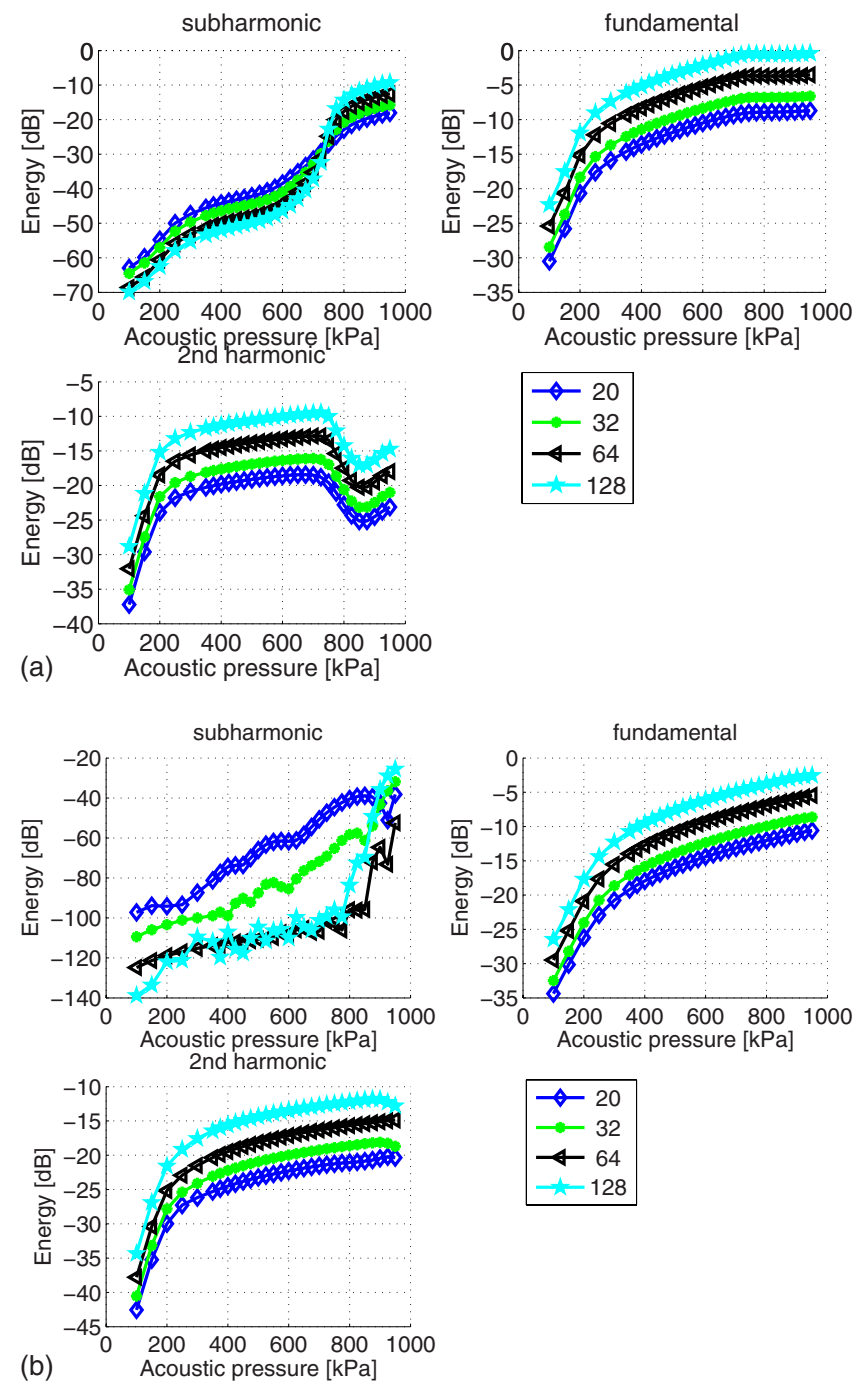

FIG. 4. (Color online) Energy as a function of acoustic pressure for Levovist. The driving pulse is a rectangular shaped (a) and a Hanning shaped (b) sinusoid, respectively. Upper left graph shows the subharmonic behavior, upper right shows the first harmonic, and lower left presents the behavior of the second harmonic component. Each curve represents a different number of cycles in the driving pulse, as displayed in the legend to the lower right in the figure.

mental, on the other hand, does not seem to be affected much although the small drop in energy observed for the rectangular driving pulse is hardly visible anymore.

The results for the simulations of Levovist as a function of acoustic pressure using a rectangular driving pulse are shown in Fig. 4(a). Once again, the three stage behavior of the subharmonic component is observed. However, now the growth stage occurs in the interval from 600 to $900 \mathrm{kPa}$. Although the increase in energy is the same, the interval is at much higher acoustic levels than experimental results achieved by Shi et al., ${ }^{10}$ who observed it to be between 300 and $600 \mathrm{kPa}$ for a custom designed (in vitro batch) suspension of Levovist using a 64 cycle rectangular driving pulse with a center frequency of $2.0 \mathrm{MHz}$. Possible reasons for this deviation can be the selection of the shell parameters itself, the difference in UCA suspension when determining the shell parameters, or the different excitation frequencies used. The fundamental and second harmonics more closely resemble 

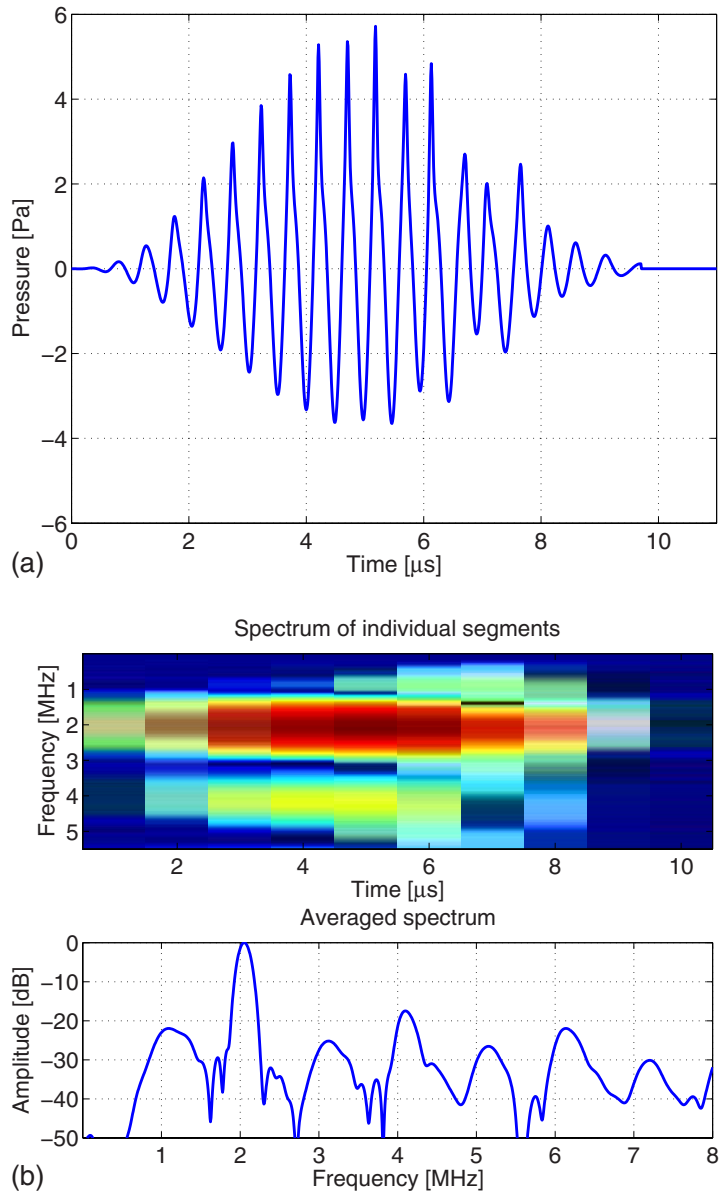

FIG. 5. (Color online) Example of scattered pressure (a) and its corresponding spectrum (b) when using a Hanning shaped driving pulse. Sonazoid is used and the excitation is a 20 cycle Hanning shaped signal with a center frequency of $2 \mathrm{MHz}$ and an acoustic pressure of $525 \mathrm{kPa}$.

the obtained measurement results. The simulations of Levovist using a Hanning shaped excitation pulse indicate that it is very hard to generate the subharmonic component for this type of driving pulse, see Fig. 4(b). In fact, the subharmonic component is hardly visible in any of the spectra, not even at the very high driving pressures. Regarding the fundamental and second harmonic components, they are similar to what was observed using the rectangular driving pulse.

Except, possibly, for the last setup, common for all the simulations is that the subharmonic component has a threshold and is present only above a certain acoustic pressure. This observation was also reported by Prosperetti ${ }^{32}$ who examined this experimentally on free bubbles and, as mentioned, by Shi et al. ${ }^{10}$ In contrast to the threshold behavior of the subharmonic, the higher harmonics seem to be present to various degrees for all driving pressures.

Finally, one interesting observation regarding the scattered pressure, when using the Hanning shaped driving pulse for excitation of Sonazoid, should be noted. When the driving pressure is increased to a level where the subharmonic is generated, the scattered response suddenly changes characteristics halfway in the pulse, as shown in Fig. 5(a). In the first half, the traditional harmonic distortion is clearly observed but no subharmonics. Halfway in the signal, the characteristic oscillation at twice the driving period is seen and
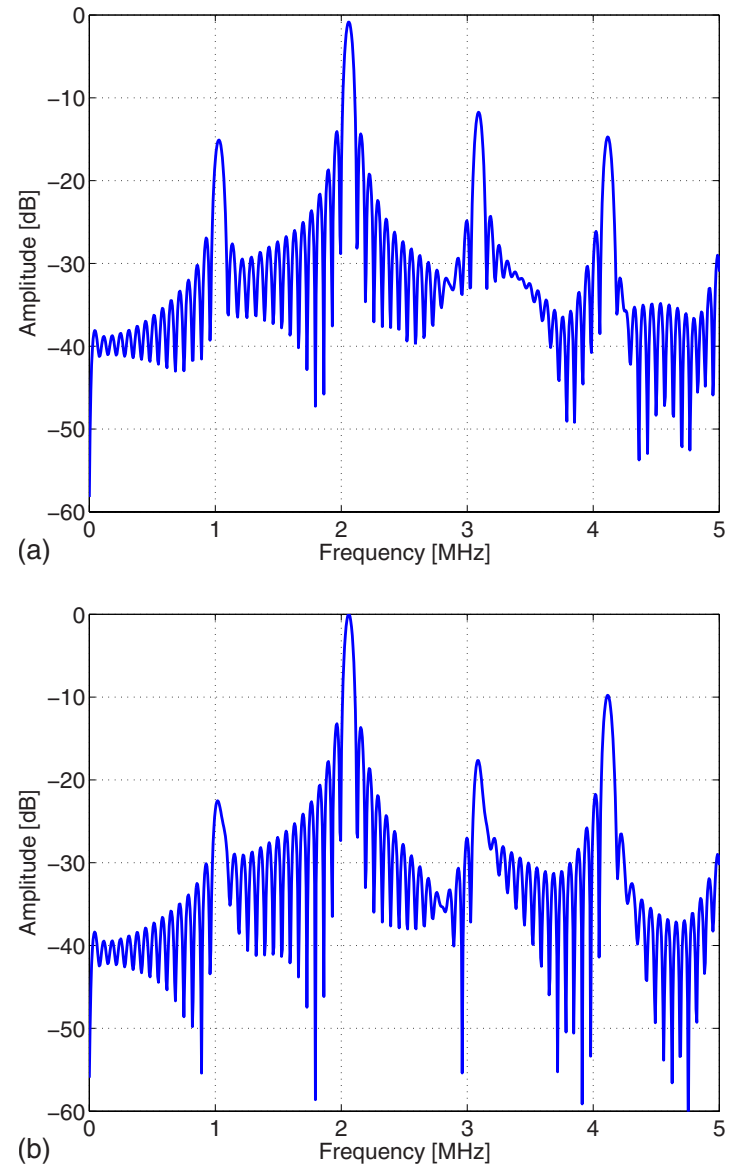

FIG. 6. (Color online) Example of spectrum of scattered response from excitation of microbubble corresponding to Levovist. The driving pulse is a 32 cycle rectangular shaped signal with a center frequency of $f_{c}$ $=2.06 \mathrm{MHz}$ and an acoustic pressure of $P_{\mathrm{ac}}=800 \mathrm{kPa}$. (a) is when no overpressure is applied and (b) shows the response when an overpressure of 25 $\mathrm{kPa}$ is applied.

continues for the rest of the scattered response. As can be seen from the corresponding spectrum in Fig. 5(b), this is what gives rise to the sub- and ultraharmonics.

\section{B. Dependence of overpressure}

In this section, the simulation results achieved when changing the ambient overpressure will be shown. Figure 6 shows an example of how the scattered spectrum changes when the ambient pressure is the only parameter that is changed from one simulation to another. The example is for Levovist when driven by a rectangular pulse with 32 cycles and an acoustic pressure of $800 \mathrm{kPa}$. In Fig. 6(a), the scattered spectrum is shown when no pressure is seen and Fig. 6(b) shows the spectrum when an overpressure of $25 \mathrm{kPa}$ is applied. Comparing the two spectra, a clear reduction in the subharmonic component at $1 \mathrm{MHz}$ is observed. No theoretical explanation for the subharmonic sensitivity to the surrounding pressure has yet been presented. However, it is likely to believe that increased damping and, perhaps, microbubble stiffness are the main mechanisms for this property as they control how the bubble oscillates. ${ }^{32}$

Figure 7 shows the effect on the subharmonic component when the pulse length is varied. It displays the energy of the subharmonic component when using the same setup as 


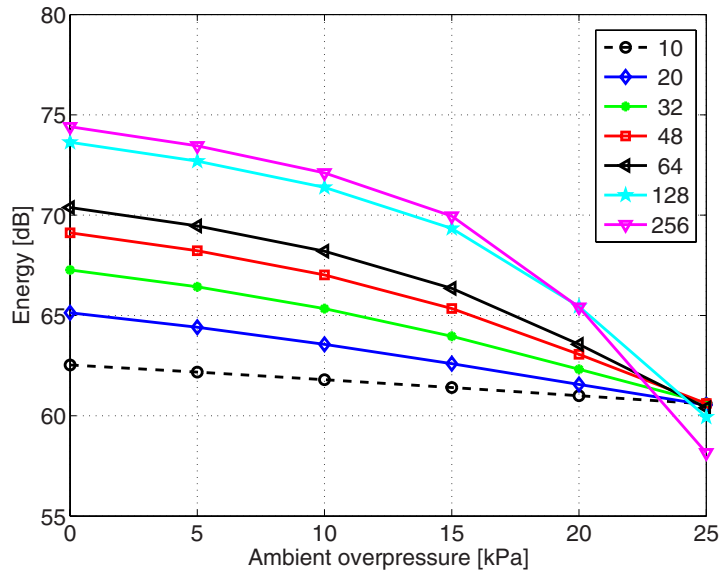

FIG. 7. (Color online) Energy of the subharmonic component scattered by Levovist when using a rectangular shaped driving pulse with an acoustic pressure of $800 \mathrm{kPa}$. The energy is displayed as a function of ambient pressure, and each curve in the plot represents a different number of cycles in the driving pulse, as indicated by the legend.

used to create Fig. 6. There is a clear tendency for all pulse lengths that the energy decreases as the overpressure is increased. Furthermore, the total decrease in energy also seems to be dependent on the number of cycles in the driving pulse. An explanation for this is probably found having Figs. 2-4, which displayed the subharmonic response simulated at $0 \mathrm{~Pa}$, in mind. These indicated that a longer driving pulse generates more subharmonic energy. However, when the ambient pressure is increased to $25 \mathrm{kPa}$, the bubble is compressed to a degree that hardly allows any subharmonic oscillations. This theory is corroborated when looking at the last ambient pressure setting in Fig. 7, indicating almost the same amount of subharmonic energy disregarding the pulse length. One final thing that should be noted from Fig. 7 is that it also indicates that the decrease is not completely linear in all cases. For easy comparison of the change in energy for the different simulation setups, Fig. 8 shows the energy of the three frequency components as function of ambient overpressure when each simulation has been normalized to their respective maximum. Looking at the results for the fundamental, it is seen that this component is not affected by ambient pressure changes. The second harmonic seems to be affected and increases about $5 \mathrm{~dB}$, slightly dependent on the pulse length. This increase is quite in contradiction to the experiments by Shi et al., ${ }^{10}$ who excited Levovist in the growth stage using a 64 cycle rectangular pulse. They found that the second harmonic decreases by $1.8 \mathrm{~dB}$ over the same ambient pressure interval. The explanation for this discrepancy is not clear as the increase in second harmonic energy is a clear trend from all the simulations performed in this study. When examining the subharmonic in Fig. 8, a highly pulse length dependent decrease is observed. As the number of pulse cycles is increased, the reduction in energy also increases. However, the decrease becomes less linear as the pulse length increases. For the driving pulse with 64 cycles, a decrease of $9.9 \mathrm{~dB}$ is found. This amount of reduction is in good agreement with similar experimental results by Shi et al., ${ }^{10}$ who measured a reduction of $9.6 \mathrm{~dB}$ using a center frequency of $2 \mathrm{MHz}$ and a driving pressure of $0.39 \mathrm{MPa}$ corresponding to the subharmonic growth interval of the current batch.

Figure 9 shows the ambient pressure sensitivity of the subharmonic component when the ambient pressure is increased from 0 to $25 \mathrm{kPa}$. The sensitivity corresponds to the absolute reduction divided by 25 and is shown as a function of the acoustic pressure and number of pulse cycles. Figure 9 thereby summarizes 252 of the most promising simulations of Levovist. Furthermore, to get a measure of the linearity between the energy of the subharmonic component and the overpressure, a straight line has been fitted using linear re-
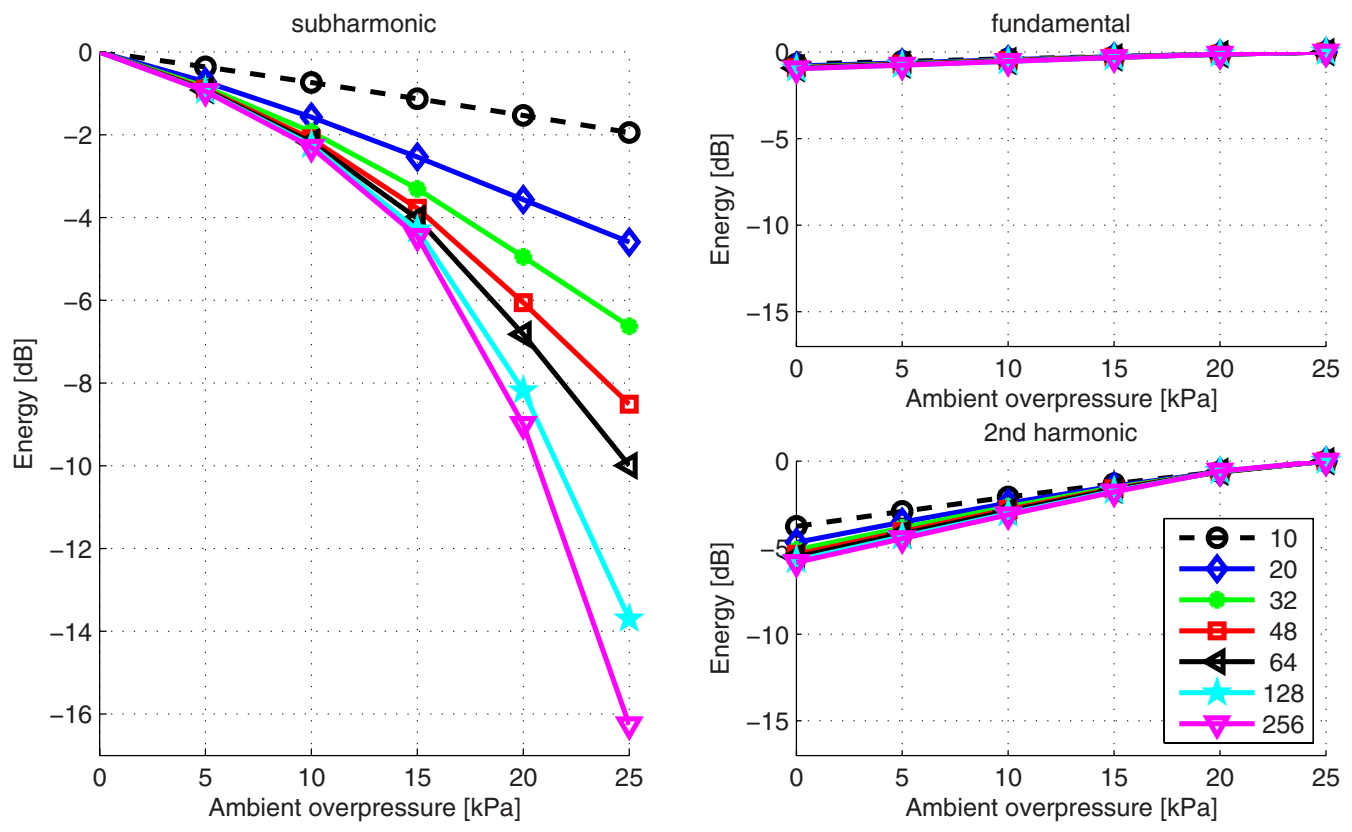

FIG. 8. (Color online) Decrease in energy of respective frequency components scattered by Levovist when using a rectangular shaped driving pulse with an acoustic pressure of $800 \mathrm{kPa}$. The energy is displayed as a function of ambient pressure, and each curve in the plots represents a different number of cycles in the driving pulse, as indicated by the legend. 

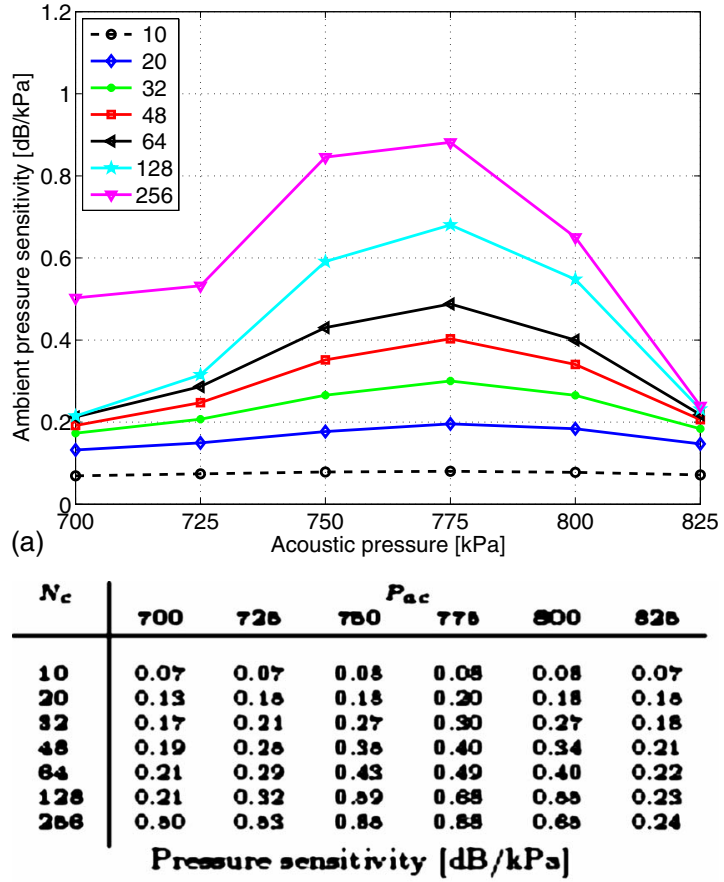

\begin{tabular}{|c|c|c|c|c|c|c|}
\hline $\boldsymbol{N}_{c}$ & 700 & 728 & 80 & $c_{778}$ & 800 & 820 \\
\hline $\begin{array}{l}10 \\
20 \\
82 \\
48 \\
64 \\
128 \\
286\end{array}$ & $\begin{array}{l}1.00 \\
1.00 \\
1.00 \\
1.00 \\
1.00 \\
0.99 \\
1.00\end{array}$ & $\begin{array}{l}1.00 \\
1.00 \\
1.00 \\
1.00 \\
0.90 \\
0.90 \\
1.00\end{array}$ & $\begin{array}{l}1.00 \\
1.00 \\
0.99 \\
0.99 \\
0.99 \\
0.97 \\
0.07\end{array}$ & $\begin{array}{l}1.00 \\
1.00 \\
1.00 \\
1.00 \\
1.00 \\
0.99 \\
0.99\end{array}$ & $\begin{array}{l}1.00 \\
1.00 \\
0.99 \\
0.98 \\
0.97 \\
0.98 \\
0.99\end{array}$ & $\begin{array}{l}1.00 \\
0.90 \\
0.90 \\
0.98 \\
0.98 \\
0.97 \\
0.97\end{array}$ \\
\hline
\end{tabular}

FIG. 9. (Color online) Ambient pressure sensitivity of the subharmonic component for Levovist when the ambient pressure is increased from 0 to 25 $\mathrm{kPa}$. The sensitivity is shown as a function of acoustic pressure and number of cycles in the rectangular driving pressure. To the right, the respective correlation coefficients, when using a linear regression model, are shown.

gression for each simulation setup when only the overpressure is changed. Next, the correlation coefficient $r$ has been calculated to see how well a linear relationship between subharmonic energy and ambient overpressure can be assumed. The respective correlation coefficients are shown to the right in Fig. 9. Figure 9 shows very clearly two characteristics: The optimal driving pressure is $775 \mathrm{kPa}$, which is in the upper end of the growth stage of the subharmonic component. Lower acoustic pressures do not generate as much subharmonic energy while higher driving pressures cause poorer sensitivity due to saturation. Furthermore, the sensitivity is increased as the driving pulse length is increased. This indicates, unfortunately, that a compromise between axial resolution and pressure sensitivity exists. The correlation coefficients to the right in Fig. 9 indicate a very good linearity. In fact, it can be seen that the two lowest coefficients are actually for the two simulations in Fig. 8 with 256 and 128 cycles, respectively. The rest of the coefficients are all equal to or above $r=0.97$. The maximum sensitivity for Levovist was achieved using a rectangular pulse of 256 cycles with a driving pressure of $775 \mathrm{kPa}$. Using this setting, a reduction in the subharmonic energy was simulated to be $22.0 \mathrm{~dB}(r$

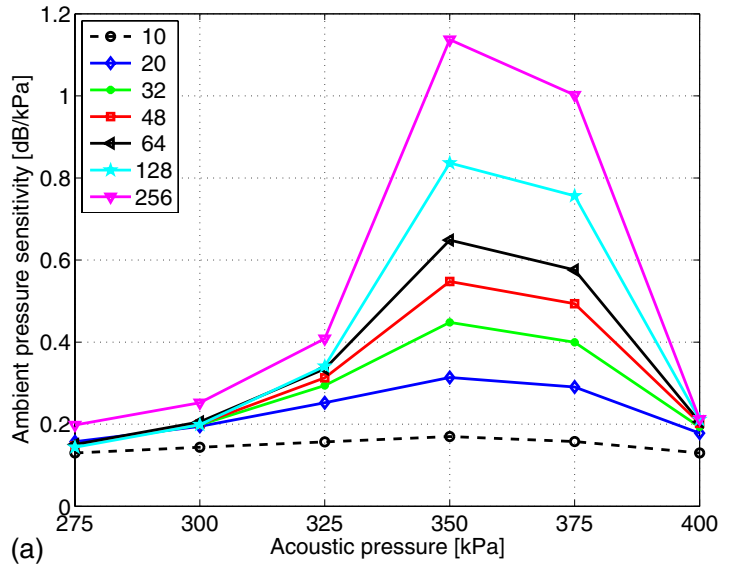

\begin{tabular}{|c|c|c|c|c|c|c|}
\hline$N_{c}$ & 278 & $\mathbf{3 0 0}$ & 328 & 500 & 970 & $\$ 00$ \\
\hline $\begin{array}{l}10 \\
20 \\
32 \\
18 \\
83 \\
128 \\
288\end{array}$ & $\begin{array}{l}0.13 \\
0.18 \\
0.18 \\
0.18 \\
0.18 \\
0.18 \\
0.20\end{array}$ & $\begin{array}{l}0.11 \\
0.12 \\
0.20 \\
0.20 \\
0.21 \\
0.20 \\
0.28\end{array}$ & $\begin{array}{l}0.16 \\
0.28 \\
0.22 \\
0.31 \\
0.33 \\
0.34 \\
0.41\end{array}$ & $\begin{array}{l}0.17 \\
0.31 \\
0.45 \\
0.85 \\
0.89 \\
0.84 \\
1.14\end{array}$ & $\begin{array}{l}0.18 \\
0.29 \\
0.40 \\
0.49 \\
0.88 \\
0.78 \\
1.00\end{array}$ & $\begin{array}{l}0.12 \\
0.18 \\
0.19 \\
0.20 \\
0.21 \\
0.21 \\
0.21\end{array}$ \\
\hline
\end{tabular}

\begin{tabular}{|c|c|c|c|c|c|c|}
\hline \multirow[t]{2}{*}{$N_{c}$} & \multicolumn{6}{|c|}{ Pac } \\
\hline & 278 & 300 & 320 & 300 & 378 & $\$ 00$ \\
\hline 10 & 1.00 & 1.00 & 1.00 & 1.00 & 1.00 & 1.00 \\
\hline 20 & 0.20 & 1.00 & 1.00 & 1.00 & 0.29 & 0.29 \\
\hline 32 & 1.00 & 0.20 & 0.00 & 0.90 & 0.28 & 0.29 \\
\hline 48 & 1.00 & 0.20 & $0 . \infty 0$ & 0.90 & 0.94 & 0.29 \\
\hline B. & 1.00 & 0.29 & 0.29 & 0.90 & 0.28 & 0.28 \\
\hline 128 & 1.00 & 0.29 & 0.28 & 0.94 & 0.24 & 0.28 \\
\hline $2 B 8$ & 1.00 & 1.00 & 0.29 & 0.98 & 0.20 & 0.08 \\
\hline
\end{tabular}

FIG. 10. (Color online) Ambient pressure sensitivity of the subharmonic component for Sonazoid when the ambient pressure is increased from 0 to $25 \mathrm{kPa}$. It is shown as a function of acoustic pressure and number of cycles in the rectangular driving pressure.

$=0.99$ ), giving a pressure sensitivity of $0.88 \mathrm{~dB} / \mathrm{kPa}$. For a shorter driving pulse with 64 cycles, the best pressure sensitivity was found to be $0.49 \mathrm{~dB} / \mathrm{kPa}(r=1.0)$.

Examining the results for Sonazoid gives the same indications as for Levovist, although the results are not as symmetric around a certain acoustic pressure. However, once again there is a clear tendency that a specific acoustic pressure in the upper end of the growth stage will optimize the ambient pressure sensitivity. Furthermore, the simulations also indicate the same relation between sensitivity and pulse length. The findings for Sonazoid are summarized in Fig. 10, which is the same as Fig. 9 for Levovist. Using a rectangular driving pulse with 256 cycles, a maximum pressure sensitivity of $1.14 \mathrm{~dB} / \mathrm{kPa}(r=0.96)$ was found. For a driving pulse with 64 cycles, the best sensitivity was found to be 0.65 $\mathrm{dB} / \mathrm{kPa}$ with a linear correlation coefficient of $r=0.99$.

\section{CONCLUSION}

A simulation study consisting of 7200 simulations has been carried out to investigate and optimize the subharmonic response sensitivity to ambient pressure changes. Two different types of ultrasound contrast agents, corresponding to Levovist and Sonazoid, were simulated. While the param- 
eters of the microbubbles were kept fixed, the parameters describing the driving pulse and ambient overpressure were changed in each simulation.

Simulations show that the subharmonic component is more easily generated using a rectangular shaped driving pulse compared to a Hanning shaped signal. For the case of Levovist, it was not possible to generate the subharmonic using the Hanning shaped excitation even for very high acoustic driving pressures. This dissimilarity in responses makes a study of the differences in shell properties of Levovist and Sonazoid interesting.

Investigations of the subharmonic energy as function of ambient overpressure showed two tendencies very clearly: The amount of reduction in energy of the subharmonic component is dependent on acoustic driving pressure and peaks when the acoustic pressure is in the upper end of the growth stage. Second, the investigations also showed a clear relation between the amount of energy reduction and length of the driving pulse. Simulations of Levovist indicate a linear change in energy of the subharmonic component as a function of ambient overpressure. Changing the overpressure from 0 to $25 \mathrm{kPa}$ indicates pressure sensitivities of 0.49 and $0.88 \mathrm{~dB} / \mathrm{kPa}$ for a rectangular driving pulse with 64 and 256 cycles, respectively. For Sonazoid, the sensitivities were found to be 0.65 and $1.14 \mathrm{~dB} / \mathrm{kPa}$ when using the same excitation pulses as for Levovist.

\section{ACKNOWLEDGMENTS}

The authors wish to thank Lars Hoff for making BUBBLESIM publicly available. This work was supported by Grant No. 26-04-0024 from the Danish Science Foundation, the Technical University of Denmark, and by B-K Medical Aps.

${ }^{1}$ D. H. Evans, W. N. McDicken, R. Skidmore, and J. P. Woodcock, Doppler Ultrasound, Physics, Instrumentation, and Clinical Applications (Wiley, New York, 1989)

${ }^{2}$ A. C. Burton, Physiology and Biophysics of the Circulation, 2nd ed. (Year Book Medical, Chicago, IL, 1972).

${ }^{3}$ A. L. Strauss, F. J. Roth, and H. Rieger, "Noninvasive assessment of pressure gradients across iliac artery stenoses: Duplex and catheter correlative study," J. Ultrasound Med. 12(1), 17-22 (1993).

${ }^{4}$ W. M. Fairbank and M. O. Scully, "A new noninvasive technique for cardiac pressure measurements: Resonant scattering of ultrasound from bubbles," IEEE Trans. Biomed. Eng. 24, 107-110 (1977).

${ }^{5} \mathrm{~B}$. Hok, "A new approach to noninvasive manometry: Interaction between ultrasound and bubbles," Med. Biol. Eng. Comput. 19, 35-39 (1981).

${ }^{6}$ P. M. Shankar, J. Y. Chapelon, and V. L. Newhouse, "Fluid pressure measurement using bubbles insonified by two frequencies," Ultrasonics $\mathbf{2 4}$, 333-336 (1986).

${ }^{7}$ V. L. Newhouse and P. M. Shankar, "Bubble size measurements using the nonlinear mixing of two frequencies," J. Acoust. Soc. Am. 75, 1473-1477 (1984).

${ }^{8}$ A. Bouakaz, P. J. Frinking, N. de Jong, and N. Bom, "Noninvasive measurement of the hydrostatic pressure in a fluid-filled cavity based on the disappearance time of micrometer-sized free gas bubbles," Ultrasound Med. Biol. 25, 1407-1415 (1999).
${ }^{9}$ A. Bouakaz, P. J. Frinking, and N. de Jong, "Noninvasive pressure measurement using microbubble contrast agent and wavelet transforms," Proc.-IEEE Ultrason. Symp. 2, 1907-1910 (2000).

${ }^{10}$ W. T. Shi, F. Forsberg, J. S. Raichlen, and L. Needleman, "Pressure dependence of subharmonic signals from contrast microbubbles," Ultrasound Med. Biol. 25, 275-283 (1999).

${ }^{11}$ L. M. Leodore, F. Forsberg, and W. T. Shi, "In vitro pressure estimation obtained from subharmonic contrast microbubble signals," Proc.-IEEE Ultrason. Symp., 2207-2210 (2007).

${ }^{12}$ F. Forsberg, J.-B. Liu, W. T. Shi, J. Furuse, M. Shimizu, and B. B. Goldberg, "In vivo pressure estimation using subharmonic contrast microbubble signals: Proof of concept," IEEE Trans. Ultrason. Ferroelectr. Freq. Control 52, 581-583 (2005).

${ }^{13}$ D. Adam, M. Sapunar, and E. Burla, "On the relationship between encapsulated ultrasound contrast agent and pressure," Ultrasound Med. Biol. 31, 673-686 (2005).

${ }^{14} \mathrm{~K}$. S. Andersen and J. A. Jensen, "In vitro measurement of ambient pressure changes using a realistic clinical setup," Proc.-IEEE Ultrason. Symp., 1096-1099 (2008)

${ }^{15} \mathrm{~K}$. S. Andersen and J. A. Jensen, "Non-invasive estimation of blood pressure using ultrasound contrast agents," in International Congress on Ultrasonics (2009).

${ }^{16} \mathrm{~L}$. Rayleigh, "On the pressure developed in a liquid during the collapse of a spherical cavity," Philos. Mag. 34, 94-98 (1917).

${ }^{17}$ M. Minnaert, "On musical air-bubbles and the sound of running water," Philos. Mag. 16, 235-248 (1933).

${ }^{18}$ M. S. Plesset, "The dynamics of cavitation bubbles," J. Appl. Mech. 16, 277-282 (1949).

${ }^{19} \mathrm{~N}$. de Jong and L. Hoff, "Ultrasound scattering properties of albunex microspheres," Ultrasonics 31, 175-181 (1993).

${ }^{20} \mathrm{C}$. C. Church, "The effects of an elastic solid surface layer on the pulsations of gas bubbles," J. Acoust. Soc. Am. 97, 1510-1521 (1995).

${ }^{21}$ K. E. Morgan, J. S. Allen, P. A. Dayton, J. Chomas, A. Klibanov, and K. Ferrara, "Experimental and theoretical evaluation of microbubble behavior: Effect of transmitted phase and bubble size," IEEE Trans. Ultrason. Ferroelectr. Freq. Control 47, 1494-1509 (2000).

${ }^{22}$ H. J. Vos, F. Guidi, E. Boni, and P. Tortoli, "Method for microbubble characterization using primary radiation force," IEEE Trans. Ultrason. Ferroelectr. Freq. Control 54, 1333-1345 (2007).

${ }^{23}$ N. de Jong, L. Hoff, and N. Bom, "Absorption and scatter of encapsulated gas filled microspheres: Theoretical considerations and some measurements," Ultrasonics 30, 95-103 (1992).

${ }^{24}$ L. Hoff, Acoustic Characterization of Contrast Agents for Medical Ultrasound Imaging (Kluwer Academic, Dordrecht, 2001).

${ }^{25} \mathrm{~K}$. S. Andersen and J. A. Jensen, "Simulation of microbubble response to ambient pressure changes," Med. Imag. V Symp. 6920, 692016 (2008).

${ }^{26} \mathrm{~L}$. Trilling, "The collapse and rebound of a gas bubble," J. Appl. Phys. 23, 14-17 (1952).

${ }^{27}$ J. B. Keller and M. Miksis, "Bubble oscillations of large amplitude," J. Acoust. Soc. Am. 68, 628-633 (1980).

${ }^{28}$ S. Hilgenfeldt and D. Lohse, "The acoustics of diagnostic microbubbles: Dissipative effects and heat deposition," Ultrasonics 38, 99-104 (2000).

${ }^{29}$ J.-F. Yu, D. Zhang, X.-F. Gong, Y.-J. Gong, Z.-M. Zhu, and X.-M. Liu, "Frequency dependences of sound attenuation and phase velocity in suspensions containing encapsulated microbubbles," Chin. Phys. Lett. 22, 892-895 (2005).

${ }^{30}$ K. Sarkar, W. T. Shi, D. Chatterjee, and F. Forsberg, "Characterization of ultrasound contrast microbubbles using in vitro experiments and viscous and viscoelastic interface models for encapsulation," J. Acoust. Soc. Am. 118, 539-550 (2005)

${ }^{31}$ W. Lauterborn and E. Cramer, "Subharmonic route to chaos observed in acoustics," Phys. Rev. Lett. 47, 1445-1448 (1981).

${ }^{32}$ A. Prosperetti, "Application of the subharmonic threshold to the measurement of the damping of oscillating gas bubbles," J. Acoust. Soc. Am. 61, 11-16 (1977). 